\title{
Design of Battery Management System
}

\author{
Chuan-wei Zhang ${ }^{1, ~ a}$, Lin-yang $\mathrm{Li}^{2, \mathrm{~b}}$ \\ ${ }^{1-2}$ College of Mechanical Engineering, Xi'an University of Science and Technology, Xi'an Shaanxi \\ 710054, China \\ azhangcw@xust.edu.cn, ${ }^{b} 1304964201 @ q q . c o m$
}

\begin{abstract}
Keywords: Battery management system; CAN bus; Cloud platform ; BP Neural network; State of Charge estimation
\end{abstract}

Abstract: Power battery is the core component of the electric vehicle, but its performance is often restricted by equilibrium and temperature conditions. In order to solve those problems, a new type of battery management system was developed. In terms of whole structure, the master slave distributed design scheme was adopted. In aspects of hardware, the battery monitoring circuit, communication circuit and protection circuit were designed. In terms of software, the balance, temperature control strategy and the SOC estimation strategy based on cloud platform neural network were used. At the same time, the man-machine interaction interface of LABVIEW was established, which could display the battery information in real time. Finally, the electronic load was served as the load to simulate the ideal working conditions. The results show that the collected voltage and temperature information is accurate, the system response is rapid, and the SOC estimation error is controlled less than $5 \%$.

\section{Introduction}

Power battery is the energy source of the electric vehicle; it determines the performance of the whole electric vehicle ${ }^{[1-3]}$. However, because of the differences in the production process of each battery, the consistency of product is difficult to be guaranteed. In addition, too high or too low temperature will affect the overall performance of the battery ${ }^{[4]}$. Therefore, world scholars focus on developing more effective battery management system (BMS) ${ }^{[5]}$. A centralized battery management system was developed by Xutong Qiao. It could detect the information of 16 batteries, and based on the double-Calman filter method to achieve the estimation of SOC precisely ${ }^{[6]}$. Zhe Li et al studied the relationship of battery capacity, internal resistance and open circuit voltage at different temperatures, which make contribution to the study of battery thermal management ${ }^{[7]}$. Stuart $\mathrm{T}$ A designed a battery balance management system, which could achieve the balance and control of each single battery ${ }^{[8]}$. Above research shows that: in the actual situation, it is necessary to fully consider the voltage balance, temperature regulation, SOC estimation and safety protection and other issues, so as to improve the comprehensive performance of the management system.

Therefore, a battery management system was developed in this paper. The system could collect the information of the single battery, realize the voltage balance, heat management and complete the estimation of SOC, and provide some reference for the establishment of a more perfect battery management system.

\section{Overall Structure Plan of System}

This design uses the master-slave distributed design. The main control chip uses TMS320LF2407A chip, which has the advantages of low power consumption and high computational efficiency. The slave chip uses MC90S08DZ60, which has the advantage of rich resources and high cost performance ${ }^{[9]}$. The communication between chips adopt the automobile level CAN bus, the host computer uses LABVIEW to establish the PC interface, which could receive the battery information of the lower computer in real time, adjust the corresponding voltage and temperature regulating parameters. The overall structure of the system is shown in Fig.1. 


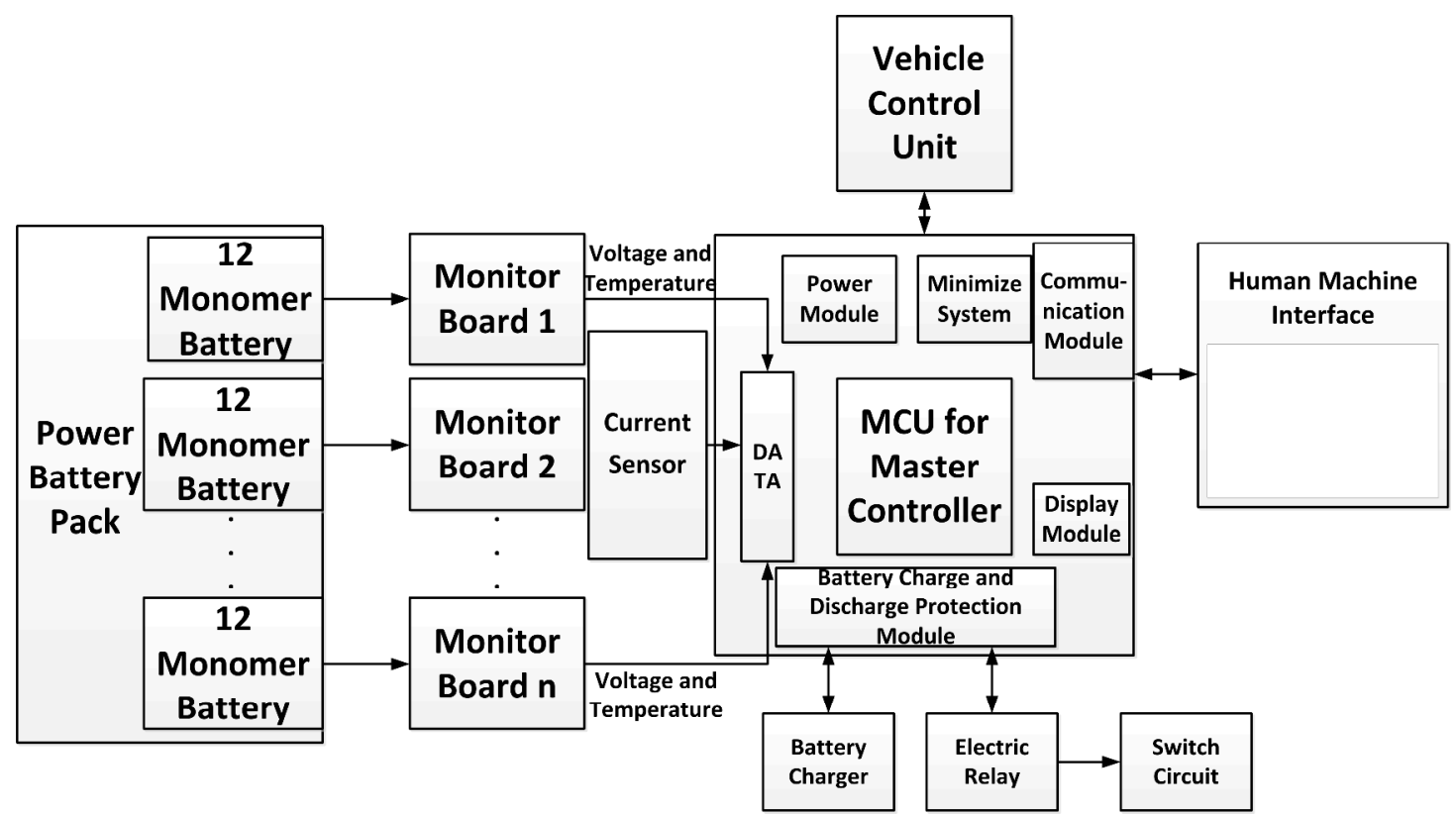

Fig. 1 Overall structure of the BMS

\section{Hardware Structures of BMS}

Single Battery Voltage Acquisition and Equalization Module. Single battery voltage information acquisition use the dedicated battery management chip LTC6804. The chip supports up to 12 battery voltage monitoring and balancing, with the advantage of high measurement speed, low error and low interference. Corresponding voltage acquisition and equalization circuit is shown in Fig. 2.

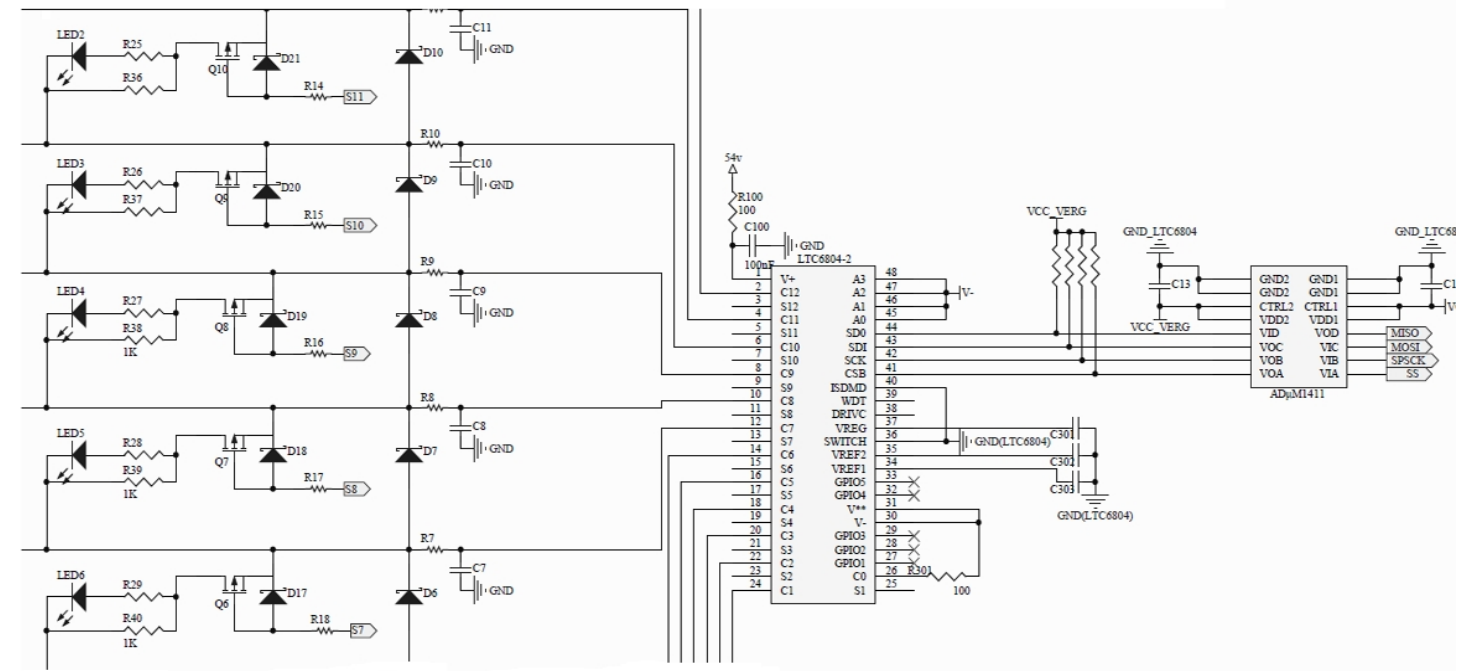

Fig. 2 Circuit of single battery voltage acquisition and equalization

The positive electrode of the monomer battery is connected to the $\mathrm{C} 1$ interface, through the RC filter circuits, finally introduced into the LTC6804 chip. Chip through the SPI communication and isolation module feed information back to the MCU of monitor board. According to the collected voltage information, MCU determine whether the need to take a balanced. If necessary, open the S port of LTC6804, turn on the MOS, consume the excess energy by resistance. At the same time, the LED lamp is lit, indicating that the balance is start.

Design of Temperature Acquisition Circuit. DS18B20 is used to collect the temperature of batteries. The sensor has the advantages of small size, high measuring precision, wide measuring range [10-11]. We make full use of the characteristics of DS18B20, six for a group, divided into two groups. Each one is attached to the surface of each individual cell, so as to accurately collect the temperature information of each battery. PRTR5VOUT2X converter provides two way $5 \mathrm{~V}$ voltage input for sensor 
group. The temperature signal output is connected with the PTD2 and PTD3 port of DZ60 respectively. The temperature signal collected by the temperature sensor could be divided into different time periods, which is helpful for the MCU of monitor board to collect the signal accurately.

Design of Power Module. The system needs a variety of power supply voltage types, just as shown in Table 1.

\begin{tabular}{cc}
\multicolumn{2}{c}{ Table 1 Different voltage types } \\
\hline Chip & Voltage(V) \\
\hline TMS320LF2407A & 3.3 \\
DZ60 & 5 \\
CAN bus & 5 \\
Touch screen & 24 \\
Hall Current Sensor & \pm 15 \\
LTC6804 & 54 \\
\hline
\end{tabular}

In order to solve the problem of multiple stable output voltage, and to eliminate the unbalance of battery capacity caused by the LTC6804 chip itself, this paper adopts the method of external power supply. Vehicle auxiliary power supply to the system input $12 \mathrm{~V}$ stable voltage, through the DC/DC, LT3954-1 chip and $15 \mathrm{~V}$ voltage conversion chip output $5 \mathrm{~V}, 24 \mathrm{~V}, 54 \mathrm{~V}$ and $+15 \mathrm{~V}$ of the voltage.

Design of Communication Module. The CAN communication module is mounted between the master and slave control board of the system, which realizes the real-time data transmitting and receiving. The main device is TJA1040. Unlike the traditional PCA82C250, this transceiver has the advantages of fast response, small electromagnetic interference, wide acceptance range, and the like. In addition, the bus also added several two channel isolation module to achieve accurate data collection. The CAN bus communication schematic diagram is shown in Fig. 3.

VIA

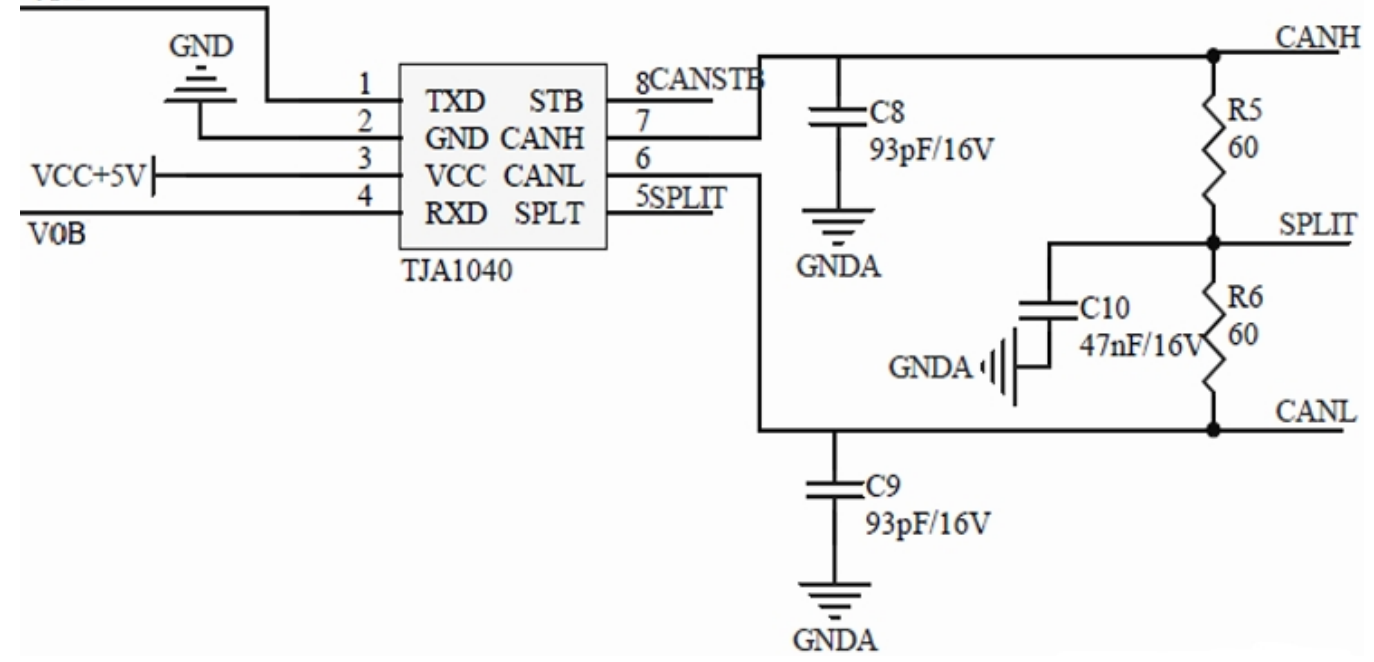

Fig. 3 CAN bus communication schematic

In order to implement communication of main control chip and PC, use the classical communication way of Serial. MAX232 chip to the next bit machine feedback signal into a 232 signal, which could be received by upper computer, so as to realize the communication between the upper and lower computer. Its schematic diagram is shown in Fig. 4. 


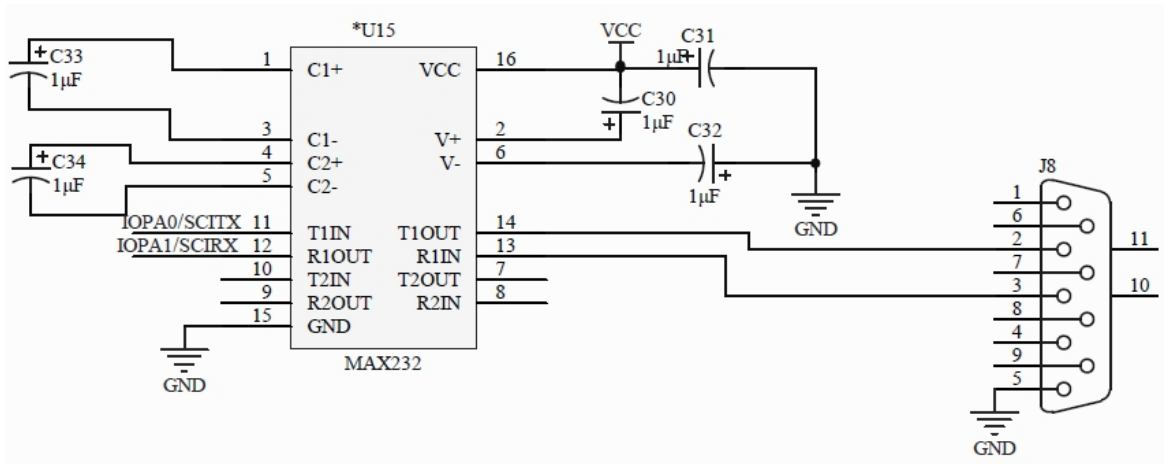

Fig. 4 Schematic of serial communication

In order to extend the flexibility of the system, it is possible to increase the support of the wireless communication interface, which provides the possibility for the realization of wireless communication.

\section{Design of System Software}

Overall structure of software. The software development of BMS is mainly based on C language, it is mainly composed of 6 parts, which are the basic information collection and SOC estimation, CAN bus communication, serial communication, balance control, temperature balance control, fault diagnosis and treatment. In order to complete information of the read \& write and drive circuit control, it also needs the assistance of clock interrupt program, the expansion of Flash cache. The general flow chart of the BMS is shown in Fig.5.

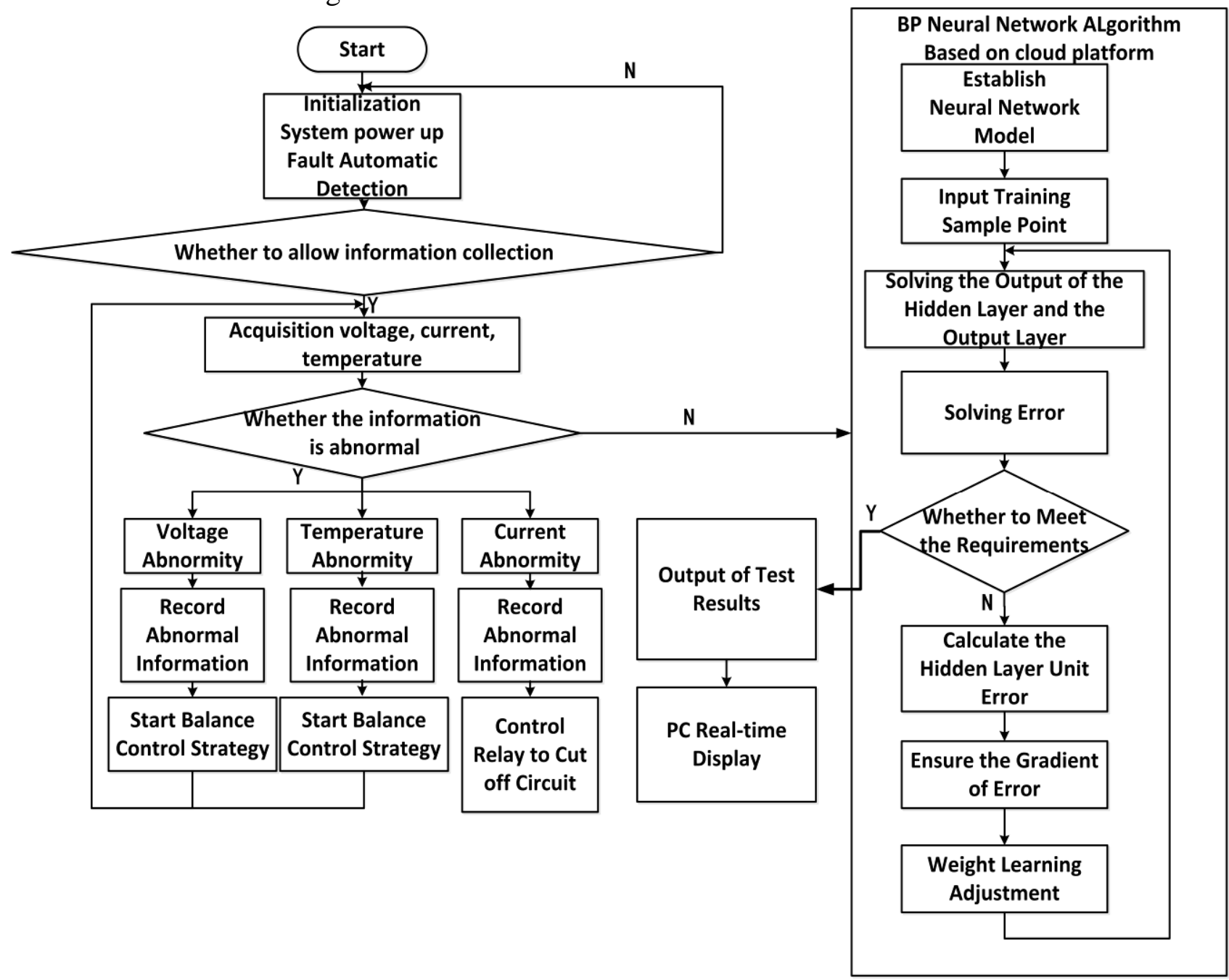

Fig. 5 Flow diagram of software system

The procedure for the implementation of the specific program could be list as follows: 
1) Initialization of the entire system: System power up and detect whether there is a fault;

2) The main program begins to run: Receive the voltage and the temperature signal from the control unit board through the CAN bus. Get the current signal through the current conditioning circuit.

3) To judge whether the temperature information is abnormal, if exceptions, interrupt the system, and then turn on the temperature control subroutine to control the corresponding drive, to achieve the temperature regulation.

4) Delay and stop interruption: According to the battery information open SOC estimation subroutine, estimate the SOC at the same time. Then write the results to the register.

5) At last, the data transmission and communication between the upper and lower computer is realized through the RS232 interface program or the wireless module communication program.

Balance and temperature control strategy. When the voltage is abnormal, in most of time, it is means that the energy of single battery is unbalanced. As is shown in Fig.6 (a), system will activate the balance control strategy.

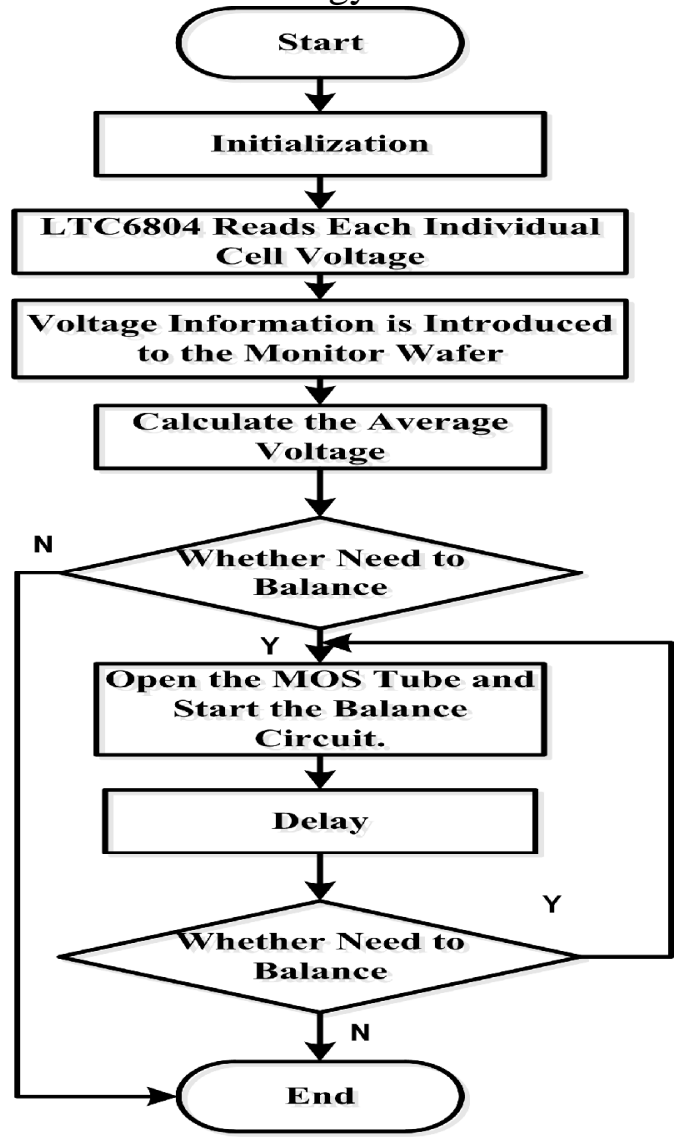

(a)

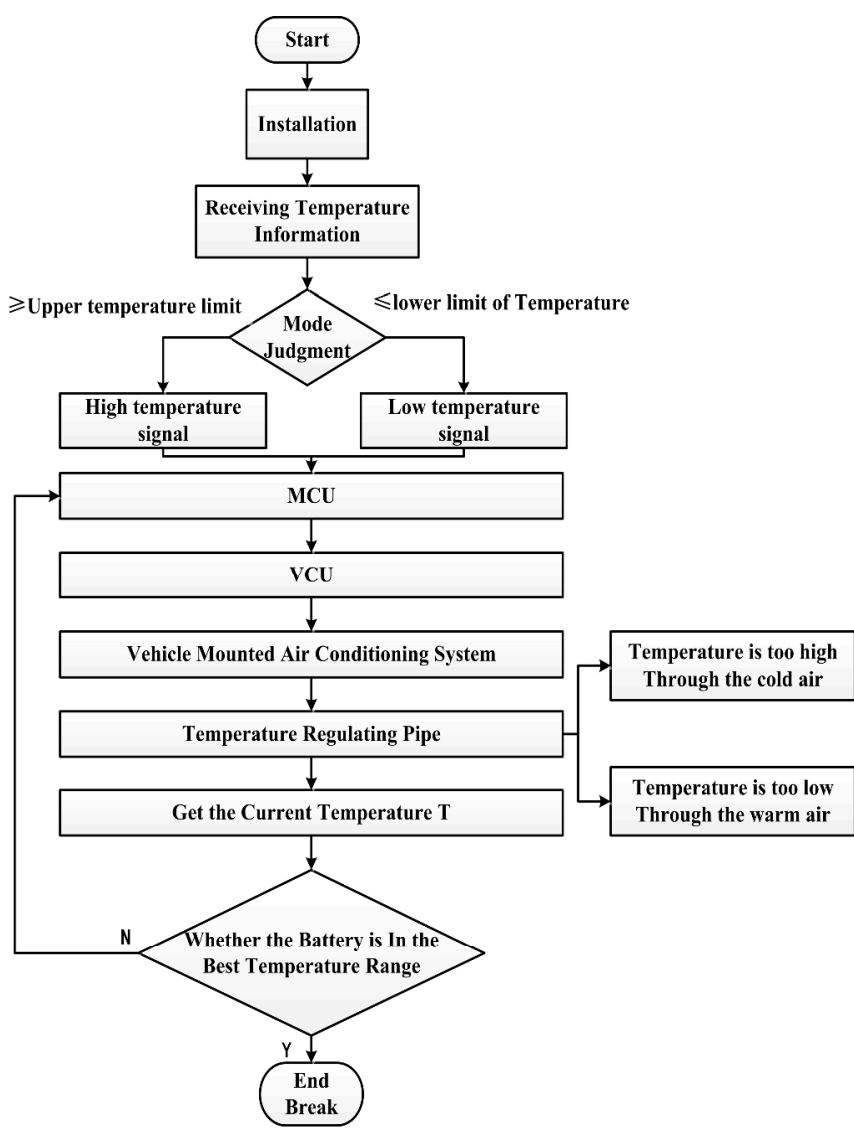

(b)

Fig. 6 Control strategy

When the temperature is abnormal, in most of time, it is means that the work temperature of batteries group is too high or too low. As is shown in Fig.6 (b), system will take the temperature control strategy.

When the current abnormal, it is likely that the system short circuit, the system into the protection mode, the main control MCU direct control relay switch circuit, cut off the overall circuit.

SOC estimation strategy. The estimation of SOC is based on BP neural network method ${ }^{[12]}$. Meanwhile, we combine the technology of cloud computing. The cloud platform contains two parts, which are database establishment and data call.

The establishment of the database also includes the establishment of neural network model and data import. The model uses the self-learning BP neural network model, which uses the classic three layer structure, including the input layer, hidden layer and output layer. Then, the battery charging and discharging test data and working condition simulation data are updated to the cloud database. These 
data are used to train the neural network model. After repeated debugging BP neural network parameters, the number of iterations, learning rate, and the training target error control value were determined. If the error of model meets the requirements, it will be built into the cloud database. In addition, the database also supports online self-learning function. The specific implementation method is that each of the battery has RFID identification tag, the battery charge and discharge test data have been recorded into the cloud platform database, which is convenient for database neural network to identify and contrast data.

Database call contains two parts, data matching and data output. Data matching contains information about the matching battery, battery management system feedback voltage, current, temperature and the like. Data output is the reference value of SOC. Finally the information is uploaded to the computer, APP and the touch screen of BMS.

\section{Experiment and Results}

In this paper, the research object is lithium iron phosphate battery for electric vehicle, rated voltage is $3.2 \mathrm{~V}$, and the capacity is $60 \mathrm{AH}$. In order to verify the accuracy of the battery management system, the battery pack with a total capacity of $72 \mathrm{~V} 120 \mathrm{AH}$ was chosen as a power supply, and the electronic load was acted as the load to simulate working conditions. The information of the battery can be monitored by the upper computer interface, just as shown in Fig .7.

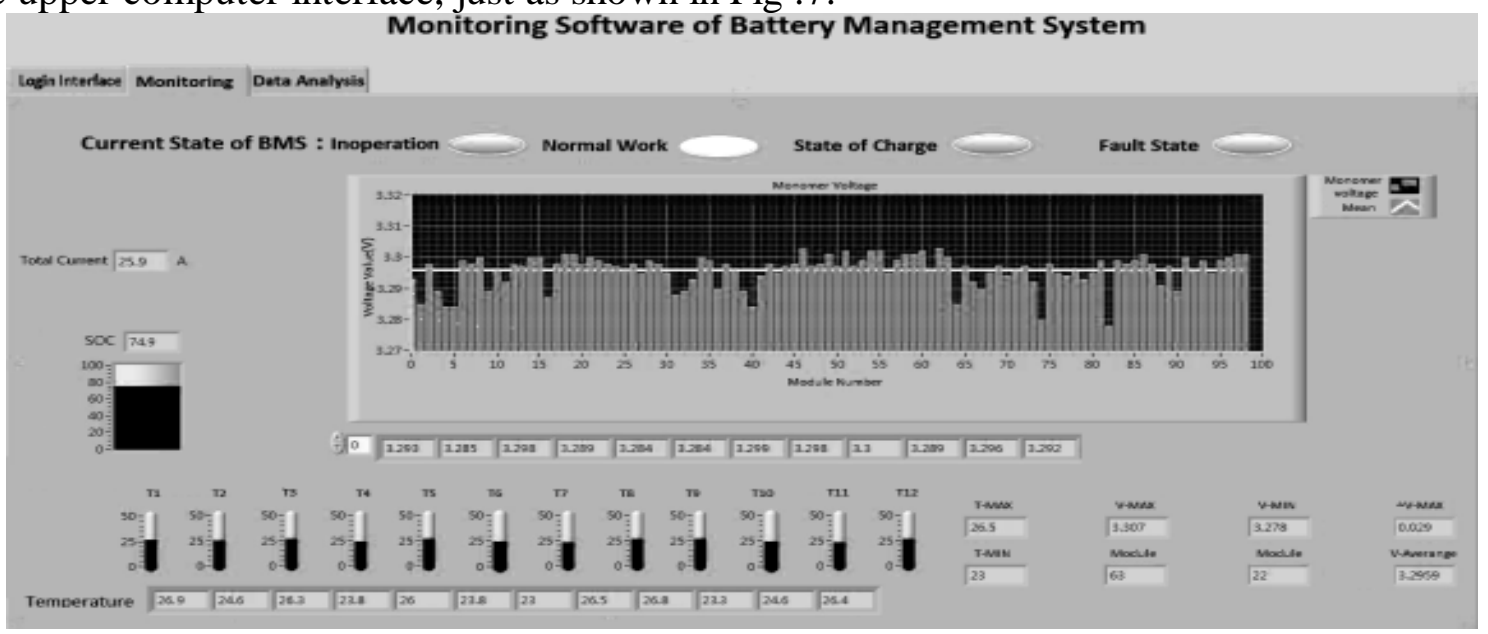

Fig.7 PC monitoring interface

According to the battery voltage information of the feedback of the upper computer, compared with the measured voltage information of the standard table, it can be seen that the voltage value of the BMS feedback and the voltage value of the standard table test are basically consistent. The results are shown in Table 2. 
Table2 Partial data of voltage measurement

\begin{tabular}{cccc}
\hline Number or battery & $\begin{array}{c}\text { Theoretical voltage } \\
(\mathrm{V})\end{array}$ & $\begin{array}{c}\text { Measured voltage } \\
(\mathrm{V})\end{array}$ & $\begin{array}{c}\text { Deviation } \\
(\mathrm{mV})\end{array}$ \\
\hline 1 & 3.290 & 3.293 & 3 \\
2 & 3.277 & 3.285 & 8 \\
3 & 3.283 & 3.298 & 15 \\
4 & 3.296 & 3.289 & -7 \\
5 & 3.296 & 3.284 & -12 \\
6 & 3.300 & 3.284 & -16 \\
7 & 3.302 & 3.299 & -3 \\
8 & 3.298 & 3.298 & 0 \\
9 & 3.298 & 3.3 & 2 \\
10 & 3.279 & 3.289 & 10 \\
11 & 3.308 & 3.296 & -12 \\
12 & 3.300 & 3.292 & -8 \\
\hline
\end{tabular}

when the battery temperature is at $0-70^{\circ} \mathrm{C}$, the temperature sampling error is controlled within $2.5^{\circ} \mathrm{C}$; When the system temperature is lower than $0^{\circ} \mathrm{C}$, there is a large deviation in the temperature measurement, which is related to the characteristics of the battery and the nature of the DS18B20.

About error estimation on SOC measurement: Electronic load provides a fixed load, which is basically equivalent to the battery to discharge $0.5 \mathrm{C}$ current test experiment. Select 225 sample time nodes in turn. The theoretical SOC of the battery are compared with the SOC values estimated by the BP neural network method.
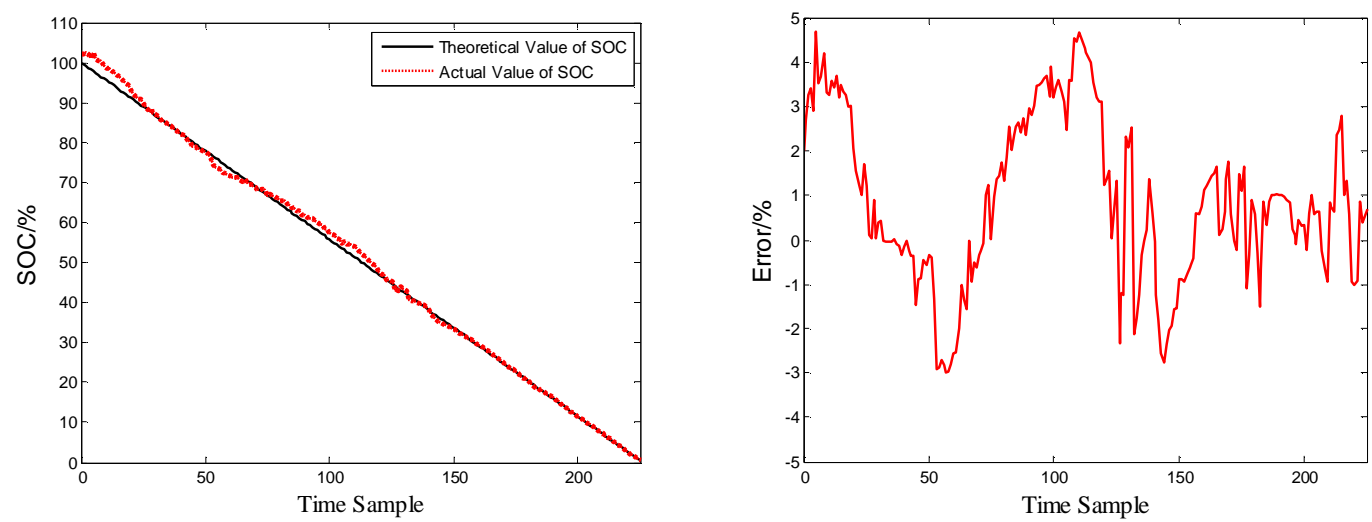

Fig.8 SOC estimation validation

As shown in Figure 8, the estimation error of SOC is less than 5\%, which meets the overall requirements of the system design.

\section{Conclusions}

The system uses a master-slave composite design ideas, support hundreds of serial battery cascade, with the advantage of stronger expansion and higher flexible.

Software and hardware platform have good compatibility, and the voltage acquisition deviation is controlled below $20 \mathrm{mV}$, the error of temperature acquisition information is less than $2.5^{\circ} \mathrm{C}$.

BP neural network method estimate the SOC, the accuracy of the estimated accuracy is less than 5\%, meet the requirements of industry standards. The system realizes the monitoring of the battery information, the balance of the single battery and the accurate estimation of the SOC.

Generally speaking, the system has good expansibility, high flexibility and high sampling precision, which provides a reliable guarantee for the safety of the power battery. 


\section{Acknowledgements}

This work was financially supported by the Science research project of Shaanxi Provincial Department of Education (11JK0869). Meanwhile, it was financially supported by Xi'an University of Science and Technology.

\section{References}

[1] Jiahao Li, J. K. Barillas, C. Guenther, et al. Multicell state estimation using variation based sequential Monte Carlo filter for automotive battery packs [J]. Elsevier Journal of Power Sources, vol. 277(2015), p. 95-103.

[2] Zhisheng An, Zhiyi Sun, Qiusheng He. Summarization of automotive Li-ion battery management system [J]. Chinese Journal of Power Sources, vol. 137, no. 6 (2013), p. 1069-1071.

[3] Languang Lu, Jianqiu Li, Jianfeng Hua, and Minggao Ouyang. Key technology of electric vehicle lithium ion battery management system [J]. Chinese Journal of Science \& Technology Review, vol. 34, no. 6 (2016), p. 39-48.

[4] Amine K, Liu J, Belharouak I. High-temperature storage and cycling of C-LiFeP04 graphite Li $\cdot$ ion cells [J]. Elsevier Journal of Electrochemistry Communications, vol. 7, no. 7 (2005), p. 669-673

[5] Xiaojun Liao, Liping He, Zhihua Zhong, et al. A review of battery management system [J]. Automotive Engineering, vol. 28, no. 10 (2006), p. 961-964.

[6] Xutong Qiao, Haifeng Geng, and Feng Dong. Design of centralized battery management system for electrical vehicle [J]. Journal of Electronic Measurement and Instrumentation, vol. 29, no. 7 (2015), p. 1019-1027.

[7] Zhe Li, Xuebin Han, and Languang Lu. Temperature characteristics of power lifepo4 batteries [J]. Journal of Mechanical Engineering, vol. 47, no. 18 (2011), p. 115-120.

[8] T. A. Start, Zhu We. Modularized bakery management for large lithium ion cells [J]. Elsevier Journal of Power Sources, vol. 196, no. 1SI (2011), p. 458-464.

[9] Yuan Jiang, Xiaowei Du, and Bojin Qi. Design of Battery Management System Based on Freescale MCU [J]. Modern Electronics Technique, vol. 34, no. 1 (2011), p. 164-166.

[10]Lei Yangyang, He Kanghua. The design of temperature measurement and control system for automatic refractometer [J]. Journal of Communications and Networking, vol. 6, no. 5 (2016), p. $20-24$.

[11]Zhihong $\mathrm{Wu}$, Gengze $\mathrm{Wu}$, Yuan $\mathrm{Zhu}$, et al. Hardware design of hybrid vehicle battery management system based on XC164CS SCM [J]. Automobile Technology, vol.4, (2009), p. $15-18$.

[12] Jin Feng. Training data selection of BP neural network for state-of-charge estimation of Li-ion battery [J]. Chinese Journal of Power Sources, vol. 140, no. 2 (2016), p. 183-186. 